\section{Perspectiva profesional y protocolos de intervención ante situaciones de maltrato hacia personas mayores}

\section{Professional perspective and intervention protocols in situations of abuse towards the elderly}

Paula Frieiro-Padín

Rubén González-Rodríguez,**

1. Doctoranda. Programa de doctorado Educación, Deporte y Salud. Universidad de Vigo (UVigo). Pontevedra. España.

2. Doctor por la Universidad de Santiago de Compostela. Profesor del Departamento de Análisis e intervención psicosocioeducativa. Universidad de Vigo (UVigo). Ourense. España.

*Autor para correspondencia.

Correo electrónico: rubgonzalez@uvigo.es (Rubén González Rodríguez).

Recibido el 30 de abril de 2019; aceptado el 28 de junio de 2019.

\section{RESUMEN}

Objetivo: Describir la situación actual de maltrato hacia las personas mayores, pormenorizando sobre los protocolos existentes en las diferentes comunidades autónomas del Estado español, así como estudiar la perspectiva de diferentes profesionales que trabajan con personas mayores y personas adultas con discapacidad. Metodología: Estudio cualitativo de revisión de documentos administrativos y protocolos de cada comunidad autónoma, así como la realización de entrevistas a 12 profesionales que trabajan directamente con personas mayores o con discapacidad en el ámbito territorial de Galicia. Paralelamente, se ha realizado una investigación de carácter documental y de revisión bibliográfica sobre el tema objeto de estudio, a través de las principales fuentes de investigación. Resultados: Los resultados reflejan la ausencia de medidas y conocimiento profesional sobre cómo intervenir ante situaciones de maltrato hacia personas mayores. Esta situación se ve agravada por la ausencia de protocolos o guías específicas en el 52,94\% de las comunidades autónomas. Conclusiones: Adicionalmente a las medidas propuestas por organismos internacionales, se considera necesario prevenir, detectar e intervenir, partiendo estas acciones de la administración pública y apoyándose en la concienciación ciudadana, de forma que se llegue a prestar una atención de calidad a la población mayor.

PALABRAS CLAVE: Maltrato, persona mayor, personas con discapacidad, protocolos, conocimiento profesional, España.

\section{ABSTRACT}

Aim: To describe the current situation of abuse towards the elderly people, detailing the existing protocols in the different autonomous communities of the Spanish State, as well as the perspective of different professionals who work with older people and disabled adults. Methodology: Qualitative review of the administrative documents and protocols of each Autonomous Community, as well as conducting interviews with 12 professionals who work directly with elderly or disabled people in the territorial area of Galicia. At the same time, a documentary research and literature review on the subject under study has been carried out through the main sources of research. Results: The results reflect the absence of measures and professional knowledge on how to intervene in situations of abuse towards elderly people. This situation is aggravated by the absence of protocols or specific guidelines in $52,94 \%$ of the autonomous communities. Conclusions: In addition to the measures proposed by international organizations, it is considered necessary to prevent, detect and intervene, and these actions of the public administration must be started and supported by citizen awareness; in such a way that we get to provide quality care to the elderly population.

KEYWORDS: Abuse, aged, disabled persons, protocols, professional knowledge, Spain.

\section{- INTRODUCCIÓN}

Existen diferentes interpretaciones sobre el maltrato a las personas mayores, muchas de ellas impulsadas por las organizaciones e iniciativas en defensa de este colectivo. Todas hacen referencia al maltrato como actos de acción u omisión, activos o pasivos, únicos o reiterados, intencionados o no (aunque no accidentales), además de a sus consecuencias. También atañen a la relación entre el causado y el causante, que ha de ser de confianza, y que, en caso de instituciones, alguna de ellas ańade la obligación legal por contrato relacional de tipo jurídico ${ }^{1}$.

Resulta destacable que no ha sido hasta el año 2002 cuando la Organización Mundial de la Salud (OMS) ha incluido el maltrato hacia las personas mayores entre sus desafíos, instando a hacerle frente a través de políticas públicas ${ }^{2}$. Por esto, y a pesar del creciente envejecimiento y a la crisis de cuidados, la sensibilización social sobre el tema todavía no está debidamente extendida.

\section{Dimensionando la realidad del maltrato a personas mayores en España}

Como todas las fuentes estadísticas apuntan, la proyección de envejecimiento poblacional se incrementará de forma progresiva. El Instituto Nacional de Estadística (INE) en 2014 apuntaba que el porcentaje de población mayor de 65 años, que en ese año se situaba en el 18,2\%, pasaría a ser del $24,9 \%$ en 2029 y del $38,7 \%$ en $2064^{3}$. De estos datos se puede desprender que la población mayor y sus características deben ser planificadas y abordadas desde el presente. Como profesionales debemos anticiparnos al futuro y repensar cuestiones como el maltrato, no pudiendo 
obviar fenómenos que afectan tan claramente a la dignidad de la persona y a los derechos humanos más esenciales.

El Instituto de Mayores y Servicios Sociales (IMSERSO) en su informe de 2016 destaca la gran heterogeneidad de estudios y datos sobre el problema, que varían en función de su procedencia ${ }^{4}$. Las estimaciones sobre el grado de extensión de los malos tratos a los mayores que se obtienen en estos estudios van desde un $0,8 \%$ de mayores de 65 años que reconocen haber sufrido al menos un tipo de maltrato, hasta un $52,6 \%$ de las personas mayores usuarias de servicios sociales o de centros de atención primaria en los que se sospecha que han sufrido malos tratos ${ }^{4}$.

En uno de los estudios de prevalencia nacional más amplios se determinó que más del $0,8 \%$ de las personas mayores entrevistadas han sido víctimas de maltrato por parte de su familia. Además, el 4,5\% de los cuidadores entrevistados declara haber maltratado al anciano bajo su cuidado en alguna ocasión en $2006^{5}$.

$\mathrm{Si}$ revisamos estudios internacionales, encontramos autores que señalan que alrededor del $25 \%$ de las personas mayores vulnerables han sufrido abusos, totalizándose así un $6 \%$ de la población anciana en general ${ }^{6}$. Ante esta falta de concordancia en algunos datos, se ve necesario la elaboración de estudios adecuados y rigurosos que tengan en cuenta la prevalencia y la sospecha, siendo indiscutible la necesidad de contar con información cuantitativa y cualitativa sobre las situaciones de malos tratos?

Se considera igualmente importante que el maltrato sea tipificado y catalogado siguiendo las tipologías establecidas por diversos autores: maltrato psicológico, negligencia, abuso económico-financiero o material y abuso sexual. Por otra parte, se debe conocer también su definición e indicadores para que, de esta forma, sirva de herramienta a tener en cuenta por las personas que estén en contacto con personas mayores, tanto en el ámbito familiar como en el profesional ${ }^{8}$.

También podrían considerarse como otras formas relevantes de maltrato: a) la obstinación diagnóstica, como realización de pruebas para aumentar el conocimiento sobre una patología o su situación clínica, sin que se prevea que vaya a tener una posterior traducción en beneficios reales y b) la obstinación terapéutica, que comprende la utilización de medios desproporcionados para prolongar artificialmente la vida biológica de un paciente con enfermedad irreversible o terminal?.

\section{Variables asociadas al maltrato en personas mayores y con discapacidad}

Determinar la vinculación entre variables sociodemográficas, el grado de dependencia, la funcionalidad económica, la autoestima, la pérdida de roles y el apoyo familiar puede resultar de utilidad a la hora de poner el foco en posibles formas de maltrato hacia personas mayores o personas adultas con discapacidad.

La sobrecarga de las personas cuidadoras es una variable importante sobre la que también debemos reflexionar. La Declaración de Toronto para la Prevención Global del Maltrato de las Personas Mayores ${ }^{2}$ alertó de que en el contexto familiar y/o en la unidad de cuidados es donde se recogen la mayoría de los casos de maltrato. El género, por su parte, es una variable de importancia que hay que tener en cuenta, ya que los estudios que comparan los perfiles de mujeres maltratadas en función de la edad muestran que, a mayor edad, existe una mayor dependencia hacia el agresor a nivel económico, físico y emocional ${ }^{10}$. Por este motivo se justifica que los protocolos o guías que establezcan las comunidades autónomas (CC.AA.) atiendan a la perspectiva de género.

Como posibles variables de riesgo individuales, como ya se ha apuntado, se describe el sexo, la edad (mayores de 75 años), el grado de de- pendencia (salud deficiente, dependencia física y conductual, deterioro cognitivo y conductas del comportamiento alteradas), a lo que se podría añadir cuestiones como zona de residencia (rural o urbana) y el tipo de vinculación familiar o profesional con la persona mayor ${ }^{11}$.

La violencia hacia las personas mayores y con discapacidad es una realidad y no responde a un único perfil, ya que estas situaciones pueden afectar a toda la sociedad en su conjunto y darse independientemente del nivel formativo o el nivel económico. Adicionalmente, se debe destacar que existen múltiples formas de maltrato (psicológico, físico, negligencia, económico y sexual), por lo que establecer un perfil único es todavía más difícil y contraproducente, ya que las personas que fuesen excluidas del perfil de víctima podrían ser todavía más difíciles de identificar. La insistencia en determinar un perfil concreto para la víctima puede dificultar la comprensión del proceso. Resulta más eficaz estudiar los obstáculos de las personas mayores para poder terminar con una situación de abuso ${ }^{12}$.

\section{- OBJETIVOS}

El objetivo de esta disertación es describir la realidad actual del fenómeno de maltrato hacia las personas mayores, teniendo en cuenta la perspectiva profesional y los protocolos y guías de las distintas CC.AA. Además, como objetivos específicos se plantean:

1. Contextualizar y dimensionar el problema a través de las múltiples investigaciones sobre el maltrato hacia las personas mayores.

2. Conocer la opinión y narrativa de profesionales que trabajan de forma directa con personas adultas con discapacidad y mayores.

3. Analizar los protocolos y guías de actuación de cada C. A.

4. Proponer posibles líneas de intervención, fruto de las impresiones de los profesionales y de las necesidades detectadas tras la búsqueda y estudio de los documentos y directrices sobre maltrato a personas mayores de cada C.A.

\section{- METODOLOGÍA}

\section{Procedimiento}

Para la elaboración de la presente investigación se ha optado por analizar información de carácter fundamentalmente cualitativa. Por una parte, revisando los documentos y protocolos de los que se dispone en cada C. A., a través de fuentes oficiales como páginas web en materia de bienestar social y/o personas mayores. Por tanto, se trata de una revisión de carácter documental en la que los términos previamente referidos se han utilizado como criterios de búsqueda. En cuanto a la revisión de los documentos y protocolos de cada C.A., cuando los resultados obtenidos en sus páginas web no reportaban ninguna información sobre maltrato a personas mayores, se contactó con las administraciones mediante correo electrónico o telefónicamente. Por otra parte, se han realizado entrevistas a través de una metodología selectiva e intencional que consistió en una entrevista a profesionales que trabajan directamente con personas mayores o con discapacidad en el ámbito territorial de Galicia.

Para poder comprender la realidad en su totalidad, y como ya se ha apuntado, se ha optado por realizar paralelamente una prospección de carácter documental y de revisión bibliográfica sobre el tema. Las principales herramientas de búsqueda utilizadas han sido, Scopus, Web of Science, SciELO, Dialnet Plus y el propio repositorio institucional de 
Tabla 1. Características laborales de la muestra

\begin{tabular}{|c|c|c|c|}
\hline N. ${ }^{0}$ de entrevista & Profesión & Trayectoria en el sector & Tipología de centro o recurso \\
\hline E1 & Trabajadora social & 8 años & Ayuda en el hogar \\
\hline E2 & Gerente & 6 años & Centro residencial \\
\hline E3 & Trabajadora social & 7 meses & ONG. Programa de envejecimiento activo \\
\hline E4 & Enfermero & 10 años & Centro de hospitalización psiquiátrica \\
\hline E5 & Trabajadora social & 3 años & Centro residencial \\
\hline E6 & Auxiliar técnico de enfermería & 11 años & Centro de hospitalización psiquiátrica \\
\hline E7 & Médico especialista en psicogeriatría & 3 años & Centro de hospitalización psiquíátrica \\
\hline E8 & Trabajadora social y gerontóloga & 2 años & Centro residencial y de día \\
\hline E9 & Enfermera & 16 años & Centro residencial y de día \\
\hline E10 & Técnica en atención a dependientes & 1 año & Centro residencial y de día \\
\hline E11 & Técnica en atención a dependientes & 14 años & Centro residencial \\
\hline E12 & Auxiliar técnica de enfermería & 7 años & Centro de hospitalización psiquíátrica \\
\hline
\end{tabular}

la Universidad de Vigo (Investigo). A través de estas herramientas se ha acotado la búsqueda combinando las palabras clave: "Maltrato", "Persona mayor", "Personas con discapacidad", "Protocolos" y "Conocimiento profesional", y se han utilizado operadores booleanos para establecer relación y así encontrar resultados más precisos. Los idiomas de búsqueda han sido el español y el inglés y no se ha establecido acotación temporal en función de la fecha de publicación. Tras el análisis de los artículos científicos, se estudiaron y consultaron 13 de ellos, los cuales fueron tenidos en cuenta en el presente estudio. Este proceso de análisis bibliográfico se ha ubicado temporalmente en el mes de marzo de 2019.

\section{Participantes}

El contacto con los profesionales se realizó a través de correo electrónico, a partir de la selección de 45 entidades registradas en el RUEPSS*. De este contacto previo accedieron a contestar 12 personas con experiencia en el ámbito de servicios y recursos para personas mayores y con un perfil profesional variado (tabla 1). Sus discursos ofrecen una visión experta a través del análisis de sus respuestas y que, además, aportan un carácter descriptivo de la situación actual.

\section{Instrumentos}

Para que la respuesta profesional fuese ágil y accesible, se procedió a la creación de una entrevista a través de 10 preguntas abiertas, usando la plataforma web Typeform, herramienta que favoreció la interacción digital. Previamente a que se realizasen las preguntas, se informó a las personas entrevistadas sobre el anonimato de sus respuestas, presentando su consentimiento informado y respetando lo dispuesto en la Ley Orgánica del 3/2018, de 5 de diciembre, de Protección de Datos Personales y garantía de los derechos digitales ${ }^{13}$.

\footnotetext{
* RUEPSS, Registro Único de Entidades Prestadoras de Servicios Sociales: recoge la información referida a las entidades que desarrollan programas o son titulares o gestoras de centros o programas de servicios sociales, en los ámbitos de mayores, discapacidad, igualdad, infancia, menores, familia, inclusión y servicios comunitarios del territorio gallego. Accesible en: https://politicasocial.xunta.gal/XiacWeb/
}

\section{RESULTADOS}

\section{Protocolos y planes de actuación actuales}

La forma de concretar los estudios y directrices internacionales, que deben implementar las administraciones públicas, es a través de protocolos, guías, proyectos y demás documentos que materialicen la necesidad de actuación ante el maltrato hacia personas mayores y personas adultas con discapacidad.

Queremos destacar que, dentro del marco de las diferentes administraciones del Estado, la facultad para realizar dichos protocolos es a través de cada C.A. Esta cuestión viene reflejada desde la Constitución española de 1978 que determinó que, para aproximar geográficamente los servicios sociales a la ciudadanía y a sus necesidades, esta competencia podría ser asumida por las autonomías, reflejándose a través del artículo 148.20, el cual refiere que las CC.AA. podrán asumir competencias en diferentes materias, entre ellas la asistencia social ${ }^{4}$. Los estatutos de autonomía suponen el marco jurídico que garantiza los derechos de los ciudadanos en este ámbito.

Se han desarrollado varios planes de actuación, muchos de ellos a nivel internacional, con el objetivo de construir una sociedad en la que las personas mayores formen parte activa de su desarrollo. Esto se ha concretado en redes internacionales, como INPEA**, con el fin de asesorar, educar e investigar sobre la protección de los derechos de las personas mayores. Además, los malos tratos hacia las personas mayores se han incluido en la investigación europea elaborada por FUTURAGE***, con un enfoque común y coordinado, que promueve una perspectiva multidisciplinar y garantiza que, tanto las prioridades como la producción investigadora reviertan en una mejora de la calidad de vida de los ciudadanos de edad avanzada ${ }^{15-17}$.

Tras haber realizado la búsqueda pormenorizada de los protocolos de maltrato a personas mayores, guías y documentos de los que se disponen

\footnotetext{
** Red internacional para la prevención del maltrato a personas mayores.

*** FUTURAGE es un proyecto que nació en 2007 y fue desarrollado por la Comisión Europea para elaborar un mapa de la investigación europea sobre envejecimiento para los siguientes 15 años.
} 
Tabla 2. Relación de protocolos o guías sobre las situaciones de maltrato a personas mayores, según comunidad autónoma

\begin{tabular}{|c|c|c|c|c|}
\hline $\begin{array}{l}\text { Comunidad } \\
\text { autónoma }\end{array}$ & Tipo de documento & Nombre del documento & Organismo responsable & $\begin{array}{c}\text { Año de } \\
\text { publicación }\end{array}$ \\
\hline Andalucía & No disponen & & & \\
\hline Aragón & Documento infográfico & $\begin{array}{l}\text { El trato de las personas mayores. Promoción } \\
\text { del buen trato y detección de malos tratos }\end{array}$ & Instituto Aragonés de Servicios Sociales & 2017 \\
\hline Asturias & Protocolo & $\begin{array}{l}\text { Protocolo sociosanitario para la promoción } \\
\text { del buen trato y la detección e intervención ante } \\
\text { el maltrato a personas mayores }\end{array}$ & $\begin{array}{l}\text { Consejería de Servicios y Derechos } \\
\text { Sociales del Principado de Asturias }\end{array}$ & 2018 \\
\hline Islas Baleares & Pendiente de publicación & & & \\
\hline País Vasco & Protocolo & $\begin{array}{l}\text { Procedimiento de prevención y detección de malos } \\
\text { tratos físicos y económicos a personas mayores } \\
\text { en la CAPV }\end{array}$ & $\begin{array}{l}\text { Departamento de Empleo y Políticas } \\
\text { Sociales }\end{array}$ & 2015 \\
\hline Canarias & No disponen. En elaboración & & & \\
\hline Cantabria & Documento infográfico & Hablando de mayores. ¿Qué son los malos tratos? & Instituto Cántabro de Servicios Sociales & 2010 \\
\hline Castilla-La Mancha & No disponen & & & \\
\hline Castilla y León & Guía & $\begin{array}{l}\text { Guía básica para la detección y actuación ante } \\
\text { situaciones de maltrato a personas mayores: } \\
\text { la dignidad no tiene edad }\end{array}$ & $\begin{array}{l}\text { Consejería de Familia e lgualdad } \\
\text { de Oportunidades }\end{array}$ & 2008 \\
\hline Cataluña & Protocolo & $\begin{array}{l}\text { Protocolo marco y orientaciones de actuación } \\
\text { contra los maltratos a las personas mayores }\end{array}$ & $\begin{array}{l}\text { Departament de Benestar Social } \\
\text { i Família }\end{array}$ & 2012 \\
\hline Extremadura & No disponen & & & \\
\hline Galicia & Guía & $\begin{array}{l}\text { Guía de actuación coordinada contra el maltrato } \\
\text { de personas mayores o adultas con discapacidad }\end{array}$ & Consellería de Política Social & 2012 \\
\hline Madrid & No disponen & & & \\
\hline Región de Murcia & Guía & Guía de buen trato a las personas mayores & $\begin{array}{l}\text { Consejería de Familia e lgualdad } \\
\text { de Oportunidades }\end{array}$ & 2017 \\
\hline Navarra & No disponen. En elaboración & & & \\
\hline La Rioja & No disponen. En elaboración & & & \\
\hline $\begin{array}{l}\text { Comunidad } \\
\text { Valenciana }\end{array}$ & No disponen & & & \\
\hline
\end{tabular}

en las diferentes comunidades, se estimó oportuno la elaboración de la tabla 2, a través de la cual se observa que el 52,94\% de las CC.AA. no disponen de documentos de actuación en la materia. Por otra parte, también se aclara su nombre, el organismo responsable que lo elaboró y el año de publicación, con la finalidad de que puedan ser fácilmente localizados y consultados por todas aquellas personas que presenten interés en la materia.

\section{Perspectiva de los profesionales}

El personal sanitario, social y el resto de los profesionales vinculados a la vejez pueden desempeñar un papel vital para ayudar a las víctimas de maltrato. Resulta fundamental conocer las consideraciones y valoraciones sobre la detección de maltrato en las instituciones y el conocimiento sobre la Guía gallega de actuación coordinada?

A continuación, se plasman algunas de las afirmaciones más relevantes que se han desprendido de la totalidad de las respuestas que han facilitado: la necesidad de publicitar los dispositivos y recursos de apoyo; el desconocimiento de los pasos a dar y del marco jurídico; el miedo que tienen las personas mayores a comunicar malos tratos recibidos; la insuficiencia de recursos; y la poca formación sobre la materia.

En cuanto a la cuestión sobre "recursos y dispositivos", los profesionales entrevistados destacan la insuficiencia de recursos y la falta de publicidad sobre los mismos (E1, E2, E5, E7). Esgrimían argumentos tales como: "no considero los recursos existentes suficientes, creo que las personas mayores se encuentran en una situación muy vulnerable, y que los recursos contra el maltrato a personas mayores son escasos" (E1). A la escasez de recursos añadían también el desconocimiento de los existentes por falta de publicidad y formación específica sobre el tema. De este modo, apuntaban que: "la publicidad de los dispositivos existentes no es suficiente, ya que no conocía el correo electrónico ni el teléfono de comunicación de maltrato" (E7).

La toma de decisiones es otra cuestión importante que preocupa a los profesionales de atención directa a personas mayores; esto se ve reflejado en las siguientes consideraciones:

"La toma de decisiones está limitada desde la gerencia del propio centro hasta las familias. Es una situación que debería ser prioritaria si hay sospecha. El profesional debería alertar..." (E1). 
"Resulta difícil tomar decisiones cuando no existen evidencias que permitan actuar contra el personal que hubiera llevado a cabo la agresión, y sobre el cual existan sospechas fundadas, pero no evidencias claras" (E2).

"El propio miedo de las personas mayores para contar lo que les ocurre limita la toma de decisiones, tanto si el maltrato es en el núcleo familiar como institucional" (E3).

"El miedo a quedar sin trabajo y la mala profesionalidad nos limita en la toma de decisiones" (E6).

"Las actuaciones en estos casos se ven limitadas por la discordancia entre los profesionales para diagnosticar el caso como tal; la familia también es un factor importante a la hora de intervenir" (E8).

Una cuestión de relevancia sobre la que incidieron los profesionales es la necesidad de mejorar su formación sobre el problema y sobre los aspectos jurídicos, tal y como se refleja a continuación:

"La formación continua y la concienciación del personal que cuida a los pacientes es básica y urgente para que no se produzca maltrato y para que los cuidados sean mejores" (E2).

"No conozco en profundidad el marco jurídico. Y sí, creo que es muy importante dar a conocer al personal sanitario todas las herramientas disponibles para un correcto ejercicio de nuestra profesión” (E7).

"Como enfermera y ciudadana no conozco el marco legal y jurídico ante situaciones de maltrato, por lo que la difusión y formación sería importante" (E9).

Adicionalmente, a partir de sus respuestas se han obtenido resultados como que solo el $8,34 \%$ de las 12 personas entrevistadas refería conocer instrumentos de detección ante sospecha de malos tratos y únicamente el 25\% de los participantes conocían las formas de comunicación en horario no administrativo. Otro dato a destacar es el escaso conocimiento entre los profesionales de la guía gallega de actuación coordinada, manifestando conocerla solo la mitad de las personas entrevistadas (tabla 3).

Tabla 3. Conocimiento de los profesionales sobre la intervención en situaciones de maltrato a personas mayores

\begin{tabular}{|l|c|c|}
\hline \multicolumn{1}{|c|}{ Conocimiento sobre la materia } & N & $\%$ \\
\hline $\begin{array}{l}\text { Conocen la guía gallega de actuación coordinada } \\
\text { sobre maltrato a personas mayores }\end{array}$ & 6 & 50 \\
\hline $\begin{array}{l}\text { Conocen instrumentos de detección como Elder } \\
\text { Abuse Suspicion Index }\end{array}$ & 1 & 8,34 \\
\hline $\begin{array}{l}\text { Conocen comunicados de urgencia en horario no } \\
\text { administrativo (teléfono social y rexistromaltrato. } \\
\text { benestar@xunta.es) }\end{array}$ & 3 & 25 \\
\hline
\end{tabular}

\section{- DISCUSIÓN}

Tras la realización del estudio y dándole respuesta a los objetivos planteados es destacable cómo a pesar de que legislativamente el maltrato hacia las personas mayores está penado o sancionado parece que, a efectos prácticos, las normativas a nivel civil y penal no son suficientes para detectar e intervenir eficazmente. A esto se suma la carencia de directrices prácticas por parte de las CC.AA.; los resultados de la tabla 2 ilustran la inexistencia de documentos concretos y protocolos de actuación en muchas de ellas. Adicionalmente, las guías y protocolos implantados son de reciente creación, siendo el protocolo más antiguo del año 2008, lo que refleja un corto bagaje sobre la materia.

En cuanto a la contextualización, a través de diferentes investigaciones, es de relevancia cómo los resultados sobre el número de personas mayores que han sufrido maltrato puede ser muy dispar, variando en 5,2 puntos porcentuales entre los estudios internacionales y los nacionales ${ }^{5,6}$. Tal y como se ha observado, estos porcentajes pueden ser muy fluctuantes en función del estudio analizado y del contexto en el que se hayan recogido los datos, reafirmándose la necesidad de establecer pautas comunes e involucrar a los distintos agentes implicados en los cuidados gerontológicos, de forma que se recojan y se pueda disponer de datos ajustados a la realidad.

Los profesionales del ámbito social y sanitario tienen, por su contacto directo y proximidad, la oportunidad de identificar tempranamente $y$, por tanto, informar del maltrato a personas mayores. Esta tarea puede ser facilitada si se dispone de instrumentos adecuados para conseguir información suficiente y saber si la persona mayor está siendo maltratada, así como qué tipo de maltrato está experimentando. Para abordar esta cuestión se ha detectado la necesidad de que los profesionales se formen y dispongan de herramientas para mejorar la actuación ante el maltrato. La tabla 3 pone de manifiesto el bajo porcentaje de profesionales que decía conocer instrumentos de detección y las formas de comunicación en horario no administrativo. Aunque los perfiles profesionales analizados han sido variados (desde personal gerente a personal auxiliar técnico de enfermería) el discurso y preocupaciones sobre la materia ha sido similar. Es importante señalar otra barrera específica, como es la actitud profesional y social ante las personas mayores, que condicionan claramente la atención en cuanto a identificación y detección del maltrato en el mayor ${ }^{18}$.

A partir de los datos recabados se ha constatado la necesidad de establecer líneas de prevención y diagnóstico para así intervenir de forma eficaz, actuando sobre la desinformación y fomentando el uso de recursos y herramientas de detección de maltrato hacia personas mayores. Además, se ignoran las directrices jurídicas y pasos administrativos que deben darse ante una situación constatada o probable de maltrato. Para conseguir estos propósitos, los organismos públicos deben favorecer medidas formativas y actuar al respecto con medidas tales como las que se proponen en la tabla 4 .

\section{- CONCLUSIONES}

Existe una amplia argumentación para defender la implantación de protocolos o guías de maltrato, pero una de las más relevantes es detectar la situación para poder intervenir y también prevenir casos futuros. Como se ha comentado, la detección del maltrato puede resultar difícil, pero si se dispone de material técnico y conocimiento, los equipos interdisciplinares estarán mejor capacitados. Debemos reflexionar que, pese a que los profesionales de atención directa pasan muchas horas con mayores (como es el caso de los auxiliares de enfermería) en ocasiones no conocen las formas de actuación y, por tanto, muchos casos no se denunciarán de forma temprana.

Queremos señalar que las administraciones públicas deben asumir y atender a los planes de actuación y directrices internacionales, sin demora, aportando un abordaje integral de este fenómeno. Sin duda, vistos los resultados sobre los protocolos existentes, parece que no se trata de una problemática que marque la agenda de la política social actual. El contexto de crecimiento de la esperanza de vida y, consecuentemente, del envejecimiento de la población espańola sugiere la necesidad de afrontar este reto, 
Tabla 4. Líneas de actuación deseables sobre las situaciones de maltrato a personas mayores

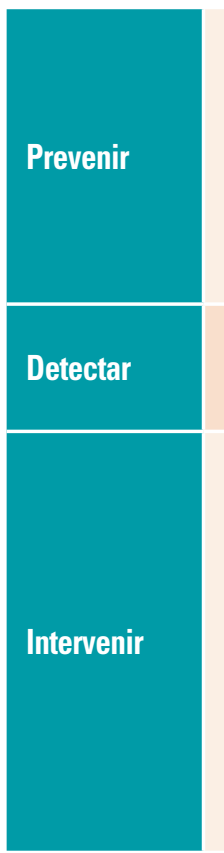

Sensibilización social del problema a través de la divulgación, campañas, guías de medidas

Ofrecer recursos como grupos de apoyo y respiros familiares para evitar sobrecarga

Formar al personal de atención directa (equipos de enfermería, terapeutas, auxiliares técnicos...)

Fomentar la investigación sobre la problemática

Formar desde las edades más tempranas (educación primaria y secundaria) sobre el proceso de envejecimiento activo y sobre los prejuicios y estereotipos asociados a esta etapa del ciclo vital

Realizar valoraciones con base en instrumentos de medición validados

Animar a las personas mayores y personas de su entorno a comunicar y denunciar, aportándoles recursos y garantías de cuidados dignos

Establecer protocolos y guías a los profesionales, de forma que puedan entender con claridad cómo actuar ante una sospecha

Creación o estandarización de un instrumento a nivel nacional para poder conseguir datos bajo un mismo criterio

Disponer de plazas de alojamiento alternativo para acoger situaciones de urgencia cerca del entorno de las personas mayores

Disponer de una red de préstamo o subvenciones para recursos técnicos que puedan mejorar la calidad de vida de la persona y cuidadores

Establecer servicios de asesoramiento y psicoterapéuticos específicos para personas mayores, y que así dispongan de orientación jurídica,

social y psicológica

Crear un registro estatal de denuncias por maltrato a personas mayores, de forma que esta realidad se pueda cuantificar con la mayor

rigurosidad posible

Impulsar normas jurídicas para agilizar las vías de incapacitación y protección para casos afectados de malos tratos que lo requieran

tanto en el marco del cuidado informal como dentro de la red existente de cuidados profesionales.

Para finalizar, queremos incidir en que la falta de datos confiables sobre el maltrato a personas mayores puede argumentar el escaso interés, prioridad y relevancia que se le otorga a este fenómeno. Por todo lo descrito anteriormente, los datos a recoger en esta materia deberían comprender la sospecha de maltrato ya que, ante circunstancias tan serias, no es necesario tener la certeza para intervenir. Actuar eficazmente ante una sospecha puede suponer terminar con la agonía de las personas mayores que sufren estas situaciones

\section{Conflicto de intereses}

Esta investigación se ha realizado sin el apoyo financiero (becas, ayudas u otro soporte económico) de ninguna institución pública o privada. No existe, por lo tanto, ningún conflicto de intereses.

\section{- BIBLIOGRAFÍA}

1. Organización Mundial de la Salud, Sociedad Española de Geriatría y Gerontología. Informe del Proyecto de colaboración (OMS IMSERSO) - Respuesta global al maltrato hacia las personas mayores, incluyendo la negligencia: capacitación de los servicios de atención primaria para el abordaje de un problema mundial. Madrid: IMSERSO; 2006.

2. Organización Mundial de la Salud [Internet]. Declaración de Toronto para la prevención global del maltrato a las personas mayores. Ginebra: OMS; 2002. [Acceso 4 de marzo de 2019]. Disponible en: http://www.who.int/ageing/en/

3. Instituto Nacional de Estadística (INE). Proyección de la población de España 2014-2064. Madrid: INE; 2014

4. Instituto de Mayores y Servicios Sociales [Internet]. Informe 2016 Las Personas Mayores en España. Datos Estadísticos Estatales y por Comunidades Autónomas. Madrid: Ministerio de Sanidad Servicios Sociales e Igualdad; 2017. [Acceso 5 de marzo de 2019]. Disponible en http://www.imserso.es/InterPresent1/groups/imserso/documents/binario/112017001_informe-2016-persona.pdf
5. Marmolejo I. Factores de riesgo del maltrato de personas mayores en la familia en población española. Zerbitzuan: Gizarte zerbitzuetarako aldizkaria. 2009;45:49-57.

6. Cooper C, Selwood A, Livingston G. The prevalence of elder abuse and neglect: a systematic review. Age Ageing. 2008:37(2):151-60.

7. Sancho M, Pérez-Rojo G, del Barrio E, Yanguas J, Izal M. Estudio de prevalencia de malos tratos a personas mayores en la Comunidad Autónoma del País Vasco. Vitoria-Gasteiz: Servicio Central de Publicaciones del Gobierno Vasco; 2011.

8. Pérez-Rojo G, Izal M, Montorio I, Nuevo R. Identificación de factores de riesgo de maltrato hacia personas mayores en el ámbito comunitario. Int J Clin Health Psychol. 2008:8(1):105-117.

9. Xunta de Galicia. Guía de actuación coordinada contra o maltrato de persoas maiores ou adultas con discapacidade. Santiago de Compostela: Secretaría Xeral de Política Social: 2011.

10. Celdrán M. La violencia hacia la mujer mayor: revisión bibliográfica Papeles del psicólogo. 2013;34(1):57-64.

11. Iborra-Marmolejo I. Maltrato de personas mayores en la familia en España. Valencia: Fundación de la Comunitat Valenciana para el estudio de la violencia de género (Centro Reina Sofía); 2008.

12. Expósito F, Moya M. Violencia de género. Mente y cerebro 2011:48:20-25

13. Ley $3 / 2018$, de 5 de diciembre, de protección de datos personales y garantía de los derechos digitales. BOE núm. 294, de 6 de diciembre de 2018.

14. Constitución Española. BOE núm. 311, de 29 de diciembre de 1978

15. Pérez-Rojo G. Chulián A. Marco conceptual de los malos tratos hacia las personas mayores. Sociedad y Utopía Revista de Ciencias Sociales. 2013;41:127-167.

16. INPEA: The International Network for the Prevention of Elder Abuse [Sede Web] [Acceso 4 de marzo de 2019]. Disponible en: http:// www.inpea.net/

17. Cordis. European Comission. FUTURAGE, a roadmap for ageing research [Sede Web] [Acceso 4 de marzo de 2019]. Disponible en: https://cordis.europa.eu/project/rcn/92038/factsheet/en

18. Torres M, Estrella I. Sensibilización y detección del maltrato en el anciano: hacia una atención primaria adaptada a los mayores. Gerokomos. 2015;26(3):79-83. 